
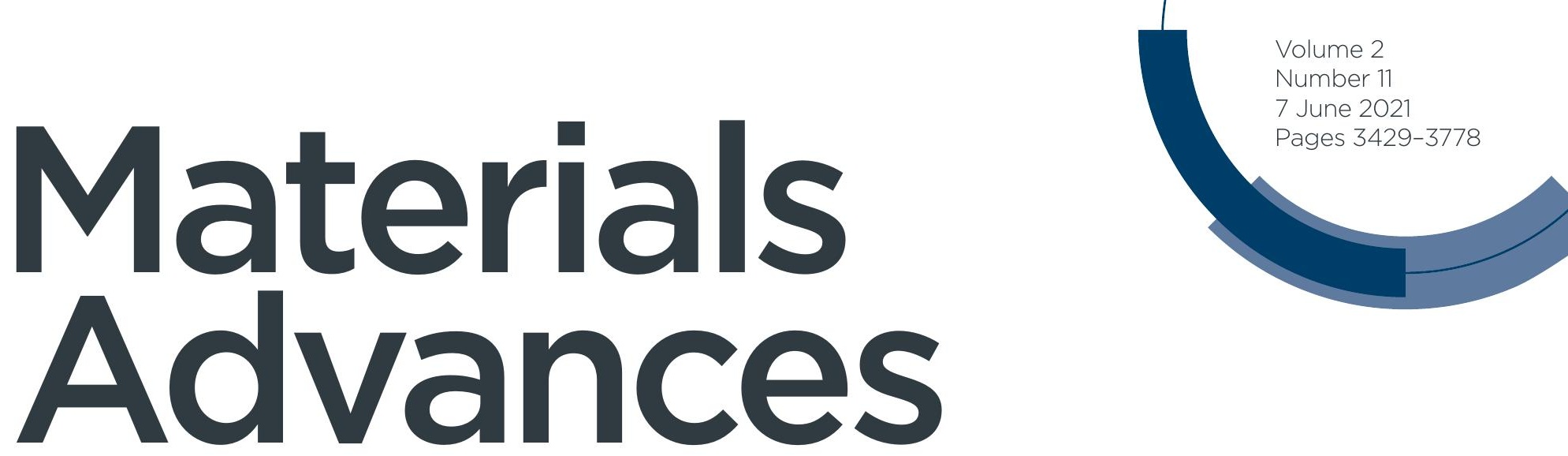

rsc.li/materials-advances

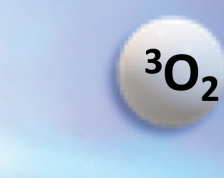

${ }^{3} \mathrm{O}_{2}$

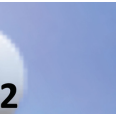


Check for updates

Cite this: Mater. Adv., 2021, 2, 3569

Received 2nd February 2021, Accepted 29th April 2021

DOI: 10.1039/d1ma00100k

rsc.li/materials-advances

\title{
Unique "posture" of rose Bengal for fabricating personal protective equipment with enhanced daylight-induced biocidal efficiency $\dagger$
}

\author{
Peixin Tang, (D) a Ahmed Y. El-Moghazy, (D) ${ }^{b}$ Bolin Ji, ${ }^{c}$ Nitin Nitin (iD ${ }^{a b}$ and \\ Gang Sun iD *a
}

\begin{abstract}
The aggregation-caused self-quenching of photosensitizers (PS), especially on a solid substrate, has highly limited their photo-induced biocidal efficiency in practical applications. Here, we designed a unique "posture" of rose Bengal (RB) on cotton-based super-adsorptive fibrous equipment, with RB being separately captured in the mesopores of porous organic polymers (POPs). The resultant daylightinduced biocidal cotton fabric with enhanced efficiency was named as DBwEE-Cotton. The enhanced biocidal activity of the DBwEE-Cotton was achieved based on two mechanisms: (1) the separation of RB in mesopores on the fabric avoids the aggregation-caused self-quenching; and (2) other than singlet oxygen generation, RB is forced to undergo type I photoreaction by surrounding the RB with massive amounts of good hydrogen donors (i.e., POP) under daylight irradiation. Given the enhanced production efficiency of reactive oxygen species by the DBwEE-Cotton, $99.9999 \%$ of $E$. coli and L. innocua bacteria were killed within 20 min of daylight exposure. The DBwEE-Cotton also presents excellent wash and light durability with no biocidal function loss. The development of DBwEE-Cotton provides a facile strategy of avoiding aggregation-caused self-quenching and modulating photoreactions of PS on a flexible substrate, which may guide the design of novel personal protective equipment (PPE) integrated with improved biocidal efficiency, wearability, and repeated and long-term applicability for protecting people from lethal infectious diseases.
\end{abstract}

\section{Introduction}

The emergence of lethal infectious diseases is always a critical threat to human health, which has been emphasized by the outbreak of the COVID-19 pandemic in 2019-2021. ${ }^{1,2}$ The availability of personal protective equipment (PPE) is crucial to protecting people, especially front-line medical workers, from cross-infection and controlling the spread of the disease. ${ }^{3,4}$ The protection mechanisms of PPE include physically blocking the contact of pathogens but without killing functions (i.e., passive protection), and biologically or chemically deactivating pathogens (i.e., aggressive protection). Mostly, traditional PPE can only passively adsorb or block the pathogens, and any live bacteria and viruses adsorbed on the PPE could cause cross-contamination

\footnotetext{
${ }^{a}$ Department of Biological and Agricultural Engineering, University of California, Davis, CA 95616, USA. E-mail: gysun@ucdavis.edu; Tel: +1 5307520840

${ }^{b}$ Department of Food Science and Technology, University of California, Davis, CA, 95616, USA

${ }^{c}$ College of Chemistry, Chemical and Biological Engineering, Donghua University, Shanghai, 201620, China

$\dagger$ Electronic supplementary information (ESI) available. See DOI: 10.1039/ d1ma00100k
}

and pose challenges during PPE recycling, disinfection, and disposal. Thus, the development of innovative materials that can aggressively attack pathogens has attracted much attention. For instance, antibacterial agents including silver nanoparticles, ${ }^{5,6} \quad \mathrm{TiO}_{2}{ }^{7} \quad N$-halamines, ${ }^{8-10}$ and organic photosensitizers ${ }^{11-14}$ were incorporated onto PPE for providing enhanced protection by disinfecting and deactivating pathogens. Among them, organic photosensitizers (PSs) (i.e., mostly are dyes) can generate reactive oxygen species (ROS), including hydroxyl radicals $\left(\mathrm{HO}^{\bullet}\right)$, hydroperoxide radicals $\left(\mathrm{HOO}^{\bullet}\right)$, hydrogen peroxides $\left(\mathrm{H}_{2} \mathrm{O}_{2}\right)$, and singlet oxygen $\left({ }^{1} \mathrm{O}_{2}\right)$, under light irradiation. The ROS are highly oxidative and lethal to microorganisms by attacking their proteins, DNA, RNA, and lipids, ${ }^{15}$ resulting in efficient and non-selective pathogen disinfection. Benzophenone derivatives were successfully grafted onto poly(vinyl alcohol-coethylene) nanofibrous membrane as a daylight-induced biocidal material. ${ }^{11}$ Moreover, benzophenone was also modified on conventional textiles, providing them with photo-induced antibacterial function without sacrificing their wearability and washability. ${ }^{16}$ Besides, the vitamin $\mathrm{K}$ family has been studied and demonstrated to be photoactive with efficient ROS production, and they were successfully incorporated into nanofibrous 
membranes to show promise for biocidal PPE design. The resultant materials performed outstanding biocidal functions against bacteria ( $E$. coli and $S$. innocua) and viruses ( $\mathrm{T} 7$ bacteriophage and coronavirus). ${ }^{12,17}$ Xanthene dyes, such as rose Bengal (RB), have been applied as antibacterial agents to functionalize textiles through the conventional dyeing process. Natural or synthetic polyamides were dyed with $\mathrm{RB}$ based on electrostatic interaction under acidic conditions. The functionalized fabrics present a moderate antibacterial function with 2-3 log of bacterial reduction within several hours of visible light irradiation. ${ }^{13,18,19}$ Recently, the introduction of poly-cationic side chains onto cotton fibers has allowed the incorporation of anionic RB on cotton fabrics effectively. ${ }^{20}$ Based on the sufficient adsorption amount of RB on the fabric and the high affinity of the cationic fabric to the negatively charged microorganisms, the material showcased a 99.9999\% reduction of E. coli, L. innocua, and T7 bacteriophage virus within $60 \mathrm{~min}$ of daylight exposure. ${ }^{20}$

Although embedding a PS on fibrous materials by chemical modification or physical adsorption brought a way for developing aggressive PPE, the biocidal efficiency of the PS can be dramatically restricted if they highly aggregated on the solid support. $^{21,22}$ For instance, the aggregation of RB molecules occurs at high concentrations even in an aqueous solution, resulting from the hydrophobic interaction between each xanthene ring. It has been reported that the aggregation of RB highly limits the light-induced ROS production via RB selfquenching, which leads to less efficient biocidal activity of the material. ${ }^{23}$ Thus, supramolecular PS, ${ }^{23}$ microcapsules, ${ }^{24}$ and self-assemble polymers ${ }^{25}$ were designed to avoid the aggregation-caused self-quenching of the PS. However, the strategies that are feasible for fibrous materials were limited in reports. $^{26}$

Herein, we designed and investigated a unique "posture" of PS on a flexible and wearable substrate by capturing PS molecules separately on cotton-based super-adsorptive fibrous equipment (SAFE-Cotton). ${ }^{27}$ With the presence of a highly porous organic polymer (POP) on the cotton fibers, it can be easily functionalized by different reagents based on an electrostatic-driven guest-host interaction (see details in Fig. S1, ESI $\dagger$ ). In this case, RB (i.e., an anionic PS) was selected to functionalize the SAFE-Cotton, thus achieving a daylightinduced biocidal cotton fabric with enhanced efficiency (i.e., DBwEE-Cotton). The capture of $\mathrm{RB}$ in the mesopores of the SAFE-Cotton is the key to enhancing the ROS production by minimizing the aggregation-caused self-quenching and modulating the photoreaction of RB.

\section{Experimental}

\subsection{Chemicals and materials}

Plain cotton fabrics Style 400 (weighting $98 \mathrm{~g} \mathrm{~m}^{-2}, 60 \times 60$ ) was purchased from TestFabrics Inc. (West Pittston, PA, USA). Rose Bengal (RB) sodium salt (dye content $\sim 60 \%$ ), cyanuric chloride (CCl), melamine, methyl iodide (MeI), and L-histidine were bought from Sigma-Aldrich (St. Louis, MO, USA).
(2-Chloro-2-hydroxypropyl)-trimethylammonium chloride (CHPTAC) was purchased from TCI (Portland, OR, USA). $N, N^{\prime}$-Dimethyl-4nitrosoaniline ( $p$-NDA) was bought from Spectrum Chemicals \& Laboratory Products (Gardena, CA, USA). All chemicals were used as received.

\subsection{Fabrication of cotton-based super-adsorptive fibrous equipment (SAFE-Cotton)}

Triazine-based highly porous organic polymers (POP) were in situ grown on cotton fibers based on our previous study. ${ }^{27}$ Specifically, six pieces of cotton fabric $\left(5 \times 5 \mathrm{~cm}^{2}\right)$ were first activated by $\mathrm{CCl}(9.8 \mathrm{mmol})$ in $100 \mathrm{~mL}$ of DMAc at $0{ }^{\circ} \mathrm{C}$ in an icewater bath for one hour. Then, the activated cotton was transferred into $90 \mathrm{~mL}$ DMSO containing $5.6 \mathrm{mmol}$ of melamine. While purging nitrogen gas into the reaction system, $30 \mathrm{~mL}$ of $\mathrm{CCl}(2.8 \mathrm{mmol})$ in DMSO was added dropwise. The reaction system was well-sealed and stirred at $500 \mathrm{rpm}$ at $150{ }^{\circ} \mathrm{C}$ for 24 hours. The resultant fabrics were washed by DMSO, deionized water $\left(\mathrm{H}_{2} \mathrm{O}\right)$, and methanol thoroughly. During $\mathrm{H}_{2} \mathrm{O}$ washing, sonication (10 $\mathrm{min}$ ) was applied to remove any weaklyadsorbed POP on the SAFE-Cotton. Finally, the SAFE-Cotton was obtained by drying the fabrics under vacuum at $30{ }^{\circ} \mathrm{C}$. The grafting ratio of POP on the SAFE-Cotton was measured by weight difference as $11.70 \%$.

\subsection{Fabrication of cationized cotton}

Cotton fabrics were cationized by CHPTAC. Firstly, three pieces of cotton fabrics $\left(5 \times 5 \mathrm{~cm}^{2}\right)$ were pre-treated by $50 \mathrm{~g} \mathrm{~L}^{-1} \mathrm{NaOH}$ solution $(50 \mathrm{~mL})$ at room temperature for $40 \mathrm{~min}$. Then, CHPTAC (60\% aqueous solution) was added into the above system to a final CHPTAC concentration of $30 \mathrm{~g} \mathrm{~L}^{-1}$. The reaction was performed at $80{ }^{\circ} \mathrm{C}$ for one hour. The resultant fabrics were washed with an excess amount of $\mathrm{H}_{2} \mathrm{O}$ and dried in an oven at $80{ }^{\circ} \mathrm{C}$ for $5 \mathrm{~min}$. The cationized cotton is denoted as CHPTAC@Cotton.

\subsection{Biocidal functionalization of cotton-based fibrous materials}

The cotton-based biocidal fibrous materials were achieved by incorporating RB onto SAFE-Cotton and CHPTAC@Cotton via a conventional dyeing process at room temperature and elevated temperature, respectively. Different initial $\mathrm{RB}$ concentrations were prepared in $\mathrm{H}_{2} \mathrm{O}$, and the $\mathrm{pH}$ of the dye solution was checked and adjusted to 5.5 if needed. One piece of SAFECotton $\left(5 \times 5 \mathrm{~cm}^{2}\right)$ was immersed in $30 \mathrm{~mL}$ of the RB solution for different durations in the dark with gentle shaking. On the other hand, one piece of CHPTAC@Cotton $\left(5 \times 5 \mathrm{~cm}^{2}\right)$ was immersed in $30 \mathrm{~mL}$ of $\mathrm{RB}$ solution at $60{ }^{\circ} \mathrm{C}$ for $10 \mathrm{~min}$ with stirring. Then, the solution was heated to $80{ }^{\circ} \mathrm{C}$ within $10 \mathrm{~min}$ and kept at $80{ }^{\circ} \mathrm{C}$ for another $30 \mathrm{~min}$. Afterward, the dyed fabrics were rinsed with $\mathrm{H}_{2} \mathrm{O}$ and dried at $80{ }^{\circ} \mathrm{C}$ for $5 \mathrm{~min}$. The adsorption amount of RB on SAFE-Cotton and CHPTAC@ Cotton was quantified by measuring the RB exhaustion by using a UV-visible (UV-vis) spectrophotometer. The calibration curve of $\mathrm{RB}$ concentration $\left(C_{\mathrm{RB}}\right.$, in a unit of $\left.\mathrm{mg}^{-1}\right)$ versus the light absorbance at $550 \mathrm{~nm}\left(A_{550}\right)$ was examined as 
$A_{550}=0.0093 \times C_{\mathrm{RB}}-0.0322, R^{2}=0.9994$. It is important to note that all the RB solutions were diluted 10 times with $\mathrm{H}_{2} \mathrm{O}$ before concentration quantification.

\subsection{Control of H-donors in the POP structure by reacting with methyl iodide (MeI)}

SAFE-Cotton was firstly functionalized by RB solution $\left(100 \mathrm{mg} \mathrm{L}^{-1}\right)$ to achieve DBwEE-Cotton ${ }_{100}$. Then, DBwEE-Cotton ${ }_{100}$ was cut to a size of $2 \mathrm{~cm} \times 2 \mathrm{~cm}$ and sealed in a $4 \mathrm{~mL}$ glass vial. Different amounts of MeI were injected into the vial and incubated under room temperature and in the dark for 24 hours. The resulting fabrics were treated under vacuum at room temperature for $60 \mathrm{~min}$ to evaporate unreacted MeI.

\subsection{Characterization}

Attenuated total reflection-Fourier transform infrared (ATR-FTIR) spectra in absorbance mode were acquired in the range of $400-4000 \mathrm{~cm}^{-1}$ with a resolution of $2 \mathrm{~cm}^{-1}$ using a Nicolet 6700 FTIR spectrometer (Thermo Fisher Scientific, MA, USA). Scanning electron microscope (SEM) images were captured using a Quattro ESEM (Thermo Fisher Scientific, MA, USA). Zeta potential was obtained with a Zetasizer Nano ZS90 (Malvern Panalytical, UK). The samples for testing zeta potential were prepared by dispersing POP particles in $\mathrm{H}_{2} \mathrm{O}$ with a concentration of $2.0 \mathrm{mg} \mathrm{L}^{-1}$. The $\mathrm{pH}$ of the POP dispersion was adjusted by $\mathrm{HCl}$ or $\mathrm{NaOH}$ to the range of 3-10. $\mathrm{N}_{2}$ adsorption-desorption isotherms were obtained from a physisorption system of ASAP 2020 (Micromeritics Co., USA). The fabric samples were cut into fine powders and de-gassed at $60{ }^{\circ} \mathrm{C}$ for 4 hours before testing. The BET surface areas and porous structures were calculated according to Brunauer-Emmett-Teller (BET) and Horvath-Kawazoe (HK) theory models, respectively. The samples for confocal laser scanning microscopy (CLSM) testing were placed on a glass slide, a drop of water was added for smoothing the fabric surface, and then a coverslip was covered on top with four-edges being sealed. Fluorescence images of the prepared fabric samples were acquired with an Olympus IX71 confocal microscope (Olympus Corporation, Tokyo, Japan) by employing an excitation wavelength of $525 \mathrm{~nm}$ and an emission wavelength of $572 \mathrm{~nm}$. Images analysis was performed using ImageJ software. MALDI-TOF-MS was acquired by a Bruker UltraFlextreme MALDI TOF/TOF mass spectrometer (MA, USA). The sample was prepared by dispersing POP in THF $\left(1 \mathrm{mg} \mathrm{mL}^{-1}\right)$ and stirred at $70{ }^{\circ} \mathrm{C}$ overnight. Then, the supernatant was transferred into a clean vial for MALDI-TOF-MS testing.

\subsection{ROS production measurement}

Reactive oxygen species, including hydroxyl radicals $\left(\mathrm{HO}^{\bullet}\right)$ and singlet oxygen $\left({ }^{1} \mathrm{O}_{2}\right)$, were measured by $p$-NDA and $p$-NDA/ L-histidine in phosphate-buffered saline (PBS, $\mathrm{pH}=7.4$ ), respectively. ${ }^{12,28}$ To avoid the physical adsorption of $p$-NDA by the fabrics, the fibrous samples were immersed in $50 \mathrm{~mL}$ of $40 \mu \mathrm{M} p$-NDA solution for 24 hours in the dark. Then, the fabric $\left(2 \times 2 \mathrm{~cm}^{2}, \sim 50 \mathrm{mg}\right)$ was immersed in $10 \mathrm{~mL}$ of $40 \mu \mathrm{M} p$-NDA solution in a glass Petri dish and exposed to daylight in an XL-1500 crosslinker for different durations for examining ROS production. The light intensity in the crosslinker was measured by a light meter (EXTECH, Model \# LT300) as 13000 lux. The color fading of the $p$-NDA solution, attributed to the quenching by hydroxyl radicals produced by the sample, was detected with a UV-vis spectrophotometer. The concentrations of $p$-NDA solution in a unit of $1 \times 10^{-5} \mathrm{M}\left(C_{p \text {-NDA }}\right)$ before and after light illumination were calculated according to a calibration curve $\left(A_{440}=0.3387 \times C_{p-\mathrm{NDA}^{-}}-0.0095, R^{2}=0.9998\right)$, and the maximum absorbance at $440 \mathrm{~nm}\left(A_{440}\right)$ was recorded. For testing the generation of ${ }^{1} \mathrm{O}_{2}$ of the sample, $0.01 \mathrm{M}$ L-histidine was added into the $p$-NDA solution. ${ }^{2}$ In this case, the decrease of the $p$-NDA concentration $\left(\Delta C_{p \text {-NDA2 }}\right)$ was attributed to the quenching of $p$-NDA by hydroxyl radicals and the singlet oxygen-oxidized L-histidine. Thus, the production of singlet oxygen can be evaluated by the difference between $\Delta C_{p \text {-NDA1 }}$ and $\Delta C_{p \text {-NDA2 }}$. It is important to note that there is no apparent color fading of the $p$-NDA solution either under dark conditions or under light but without RB-embedded fabrics.

\subsection{Antibacterial test}

The antibacterial tests were performed according to the American Association of Textile Chemists and Colorists (AATCC) 100 Test Method with modifications. All the reported results were obtained as an average in triplicate. The antibacterial function of DBwEECotton was examined against two model bacteria: Gram-negative Escherichia coli 0157:H7 [American Type Culture Collection 700728] (E. coli) and Gram-positive Listeria innocua [American Type Culture Collection 33090] (L. innocua). First, E. coli and $L$. innocua colonies were mixed individually with $10 \mathrm{~mL}$ lysogeny broth and $10 \mathrm{~mL}$ trypticase soy broth, respectively, and incubated at $37^{\circ} \mathrm{C}$ for 24 hours. Then, the bacterial culture suspension was run for two cycles of centrifugation (5000 rpm, $8 \mathrm{~min}$ ) and washing (10 mL cold PBS). After that, $10 \mathrm{~mL}$ of PBS was mixed with bacteria precipitate as the final bacterial culture suspension. Around $2 \times 10^{8} \mathrm{CFU}$ per $\mathrm{mL} E$. coli and $5 \times 10^{6}$ L. innocua cultures can be obtained for further antibacterial tests.

DBwEE-Cotton $\left(2 \times 2 \mathrm{~cm}^{2}\right)$ was placed in a Petri dish and can be completely wet by $20 \mu \mathrm{L}$ of bacterial culture suspension. Then, bacteria-contaminated fabrics were exposed to daylight in a XL-1500 crosslinker or incubated under dark conditions for different durations. Sterile PBS $(10 \mu \mathrm{L})$ was dropped on the sample surface every $5 \mathrm{~min}$ to avoid inactivation of the microorganisms from increased temperature and water evaporation during light illumination. After that, the residual bacteria on the fabric were extracted by $1 \mathrm{~mL}$ of sterile PBS and were serially diluted $\left(\times 10^{0}, \times 10^{1}, \times 10^{3}, \times 10^{5}\right)$ to be inoculated on a lysogeny agar plate or trypticase soy agar plate for E. coli and L. innocua enumeration at $37^{\circ} \mathrm{C}$ for 24 hours, respectively. The antibacterial function of the material was evaluated by the plate count of residual bacterial CFU numbers. All the bacterial reduction was calculated based on the CFU number obtained on the pristine cotton, and it showed negligible effects on the killing of bacteria either under light or dark conditions.

\subsection{Gaussian calculations}

All theoretical calculations, including ground-state structure optimization and frequency calculations, were performed with 
Gaussian 09 at the unrestricted DFT-B3LYP/LanL2DZ level of theory. $\mathrm{H}_{2} \mathrm{O}$ in the conductor-like polarizable continuum model (CPCM) was used as the solvent system. The electrostatic mapping and HOMO/LUMO orbital images were obtained based on optimized geometries and viewed by GaussView 5.0.

\section{Results and discussion}

\subsection{Fabrication of daylight-induced biocidal cotton with enhanced efficiency (DBwEE-Cotton)}

Fig. 1a displays the fabrication process of SAFE-Cotton and DBwEE-Cotton. The in situ growth of POP was accomplished by using $\mathrm{CCl}$ and melamine as precursors. After cotton activation by $\mathrm{CCl}$ in an ice-water bath for $60 \mathrm{~min}$, the SAFE-Cotton was finally obtained via a condensation reaction between melamine and CCl. After that, RB was incorporated onto the SAFE-Cotton by adsorption (i.e., dyeing at room temperature) (Fig. 1a). The SEM images visually proved the growth of mesoporous POP on the cotton fibers and showed a negligible effect on the POP morphology after RB adsorption (Fig. 1b). The optical images of the SAFE-Cotton and DBwEE-Cotton ${ }_{100}$ (the subscripted 100 refers to the initial $\mathrm{RB}$ concentration) are inserted in Fig. 1b-i and b-iii, respectively. The color changes in the fabrics were evaluated by CIELab color coordinators, whiteness index, and yellowness index, which are summarized in Table S1 (ESI $\dagger$ ). The obvious color change in the fabric from pale-yellow to shining pink illustrated the sufficient loading of RB on the DBwEECotton, which is one of the crucial factors to the biocidal activity. With the presence of POP on the SAFE-Cotton (grafting ratio $=11.70 \%$ ), it possesses improved BET surface area of $38.95 \mathrm{~m}^{2} \mathrm{~g}^{-1}$ and porosity (pore volume $=0.083 \mathrm{~mL} \mathrm{~g}^{-1}$ ), which are 19 times and 13.8 times higher than that of the pristine cotton (i.e., surface area $=2.05 \mathrm{~m}^{2} \mathrm{~g}^{-1}$; pore volume $=$ $0.006 \mathrm{~mL} \mathrm{~g}^{-1}$ ) (Fig. 1c), benefiting the RB functionalization via the guest-host adsorption. The adsorption amount of RB reached saturation (i.e., 18-19 $\mathrm{mg} \mathrm{g}^{-1}$ ) when the initial concentration was $250 \mathrm{mg} \mathrm{L}^{-1}$ or higher (Fig. S2, ESI $\dagger$ ). Then, the BET surface area and pore volume of the DBwEE-Cotton ${ }_{100}$ dropped to $6.03 \mathrm{~m}^{2} \mathrm{~g}^{-1}$ and $0.017 \mathrm{~mL} \mathrm{~g}^{-1}$ after RB adsorption, respectively. In addition, the mesopore size of SAFE-Cotton and the theoretical molecular diameter of $\mathrm{RB}$ were measured as

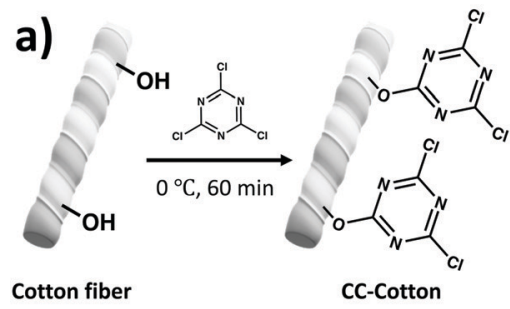

b)

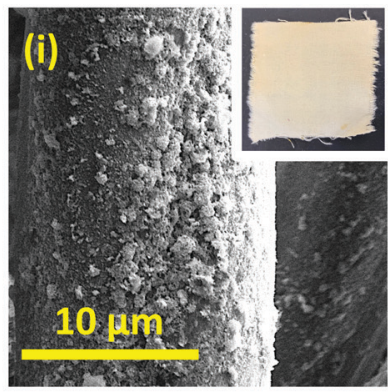

c)

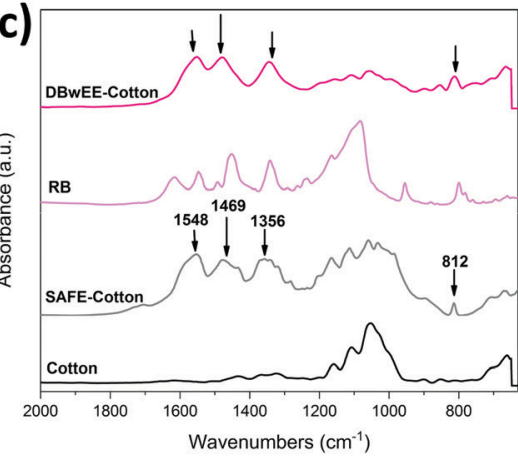

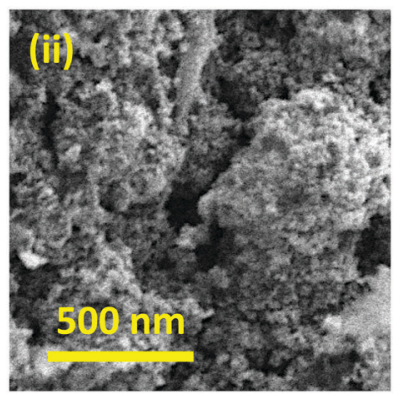

d)

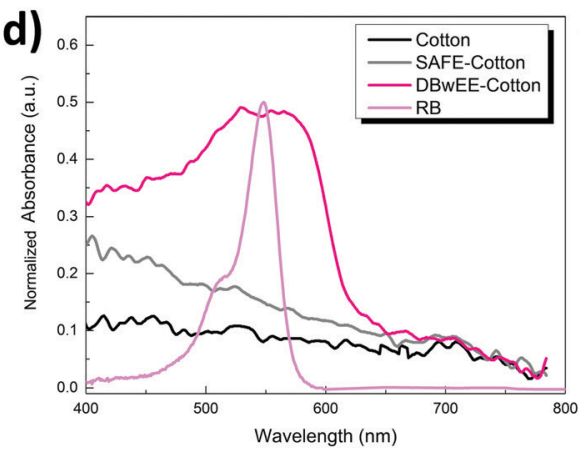

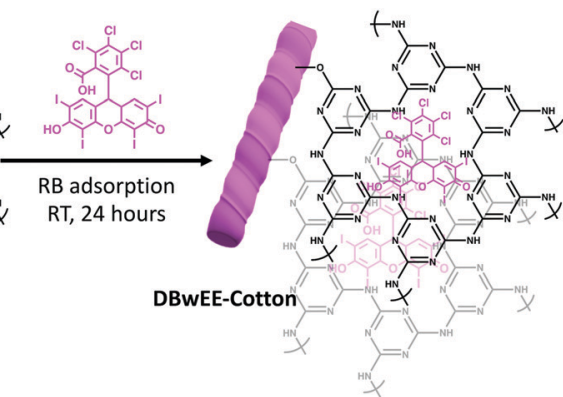
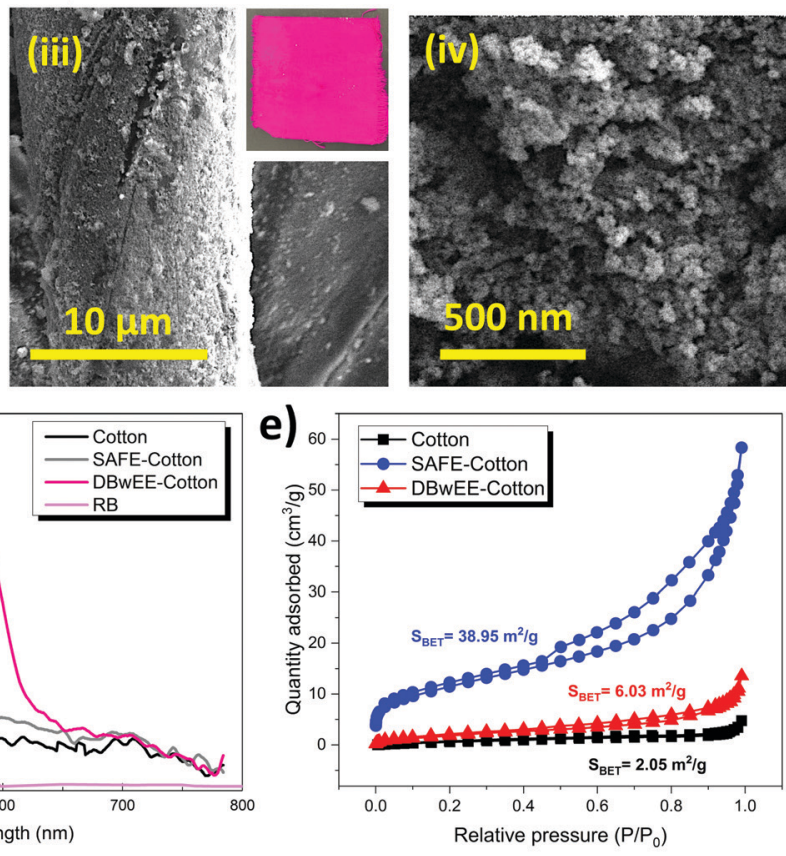

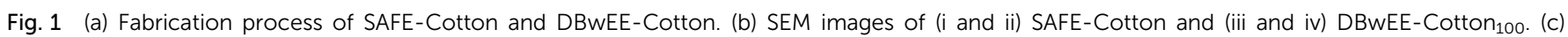
ATR-FTIR spectra, (d) diffuse reflective UV-vis spectra, and (e) $\mathrm{N}_{2}$ adsorption-desorption isotherms of relevant materials at $77 \mathrm{~K}$. 
$4.570 \mathrm{~nm}$ and around $11 \AA$ A, respectively, making RB molecules fit well in the pores of POP (Fig. S3, ESI $\dagger$ ). These results demonstrated the filling of the mesopores of the SAFE-Cotton by $\mathrm{RB}$ molecules (Fig. 1c). To understand the mathematical relationship of the $\mathrm{RB}$ capture by the $\mathrm{POP}$, the adsorption capacity of the POP particles was examined as $102.58 \mathrm{mg} \mathrm{g}^{-1}$ after 24 hours of adsorption in a $500 \mathrm{mg} \mathrm{L}^{-1} \mathrm{RB}$ solution $(\mathrm{pH}=$ 5.5). According to the MALDI-TOF-MS results of the POP particles $([\mathrm{M}+\mathrm{H}]=877.323)$, each $\mathrm{RB}$ molecule was captured in a mesopore built by 10.83 layers of POP (Fig. S4, ESI $\dagger$ ). This phenomenon further ensures the separation of RB molecules inside the POP.

Furthermore, ATR-FTIR spectra were acquired to examine the chemical structure changes in the cotton fabrics after different treatments. As presented in Fig. 1d, three broad peaks at $1548 \mathrm{~cm}^{-1}, 1469 \mathrm{~cm}^{-1}$, and $1356 \mathrm{~cm}^{-1}$ refer to the triazine ring of POP on the SAFE-Cotton. ${ }^{29}$ Moreover, a sharp peak at $812 \mathrm{~cm}^{-1}$ in the SAFE-Cotton spectrum stands for the breathing mode of the triazine ring in the POP. ${ }^{29}$ No obvious peak pattern change was found after $\mathrm{RB}$ adsorption, since most of the characteristic peaks of RB were overlapped by SAFE-Cotton. The presence of RB on the DBwEE-Cotton 100 led to a characteristic diffuse reflection UV-vis absorption peak in the range of 400$650 \mathrm{~nm}$ (Fig. 1e). Although the absorbance peak of RB on the DBwEE-Cotton $_{100}$ is much broader than that of the $\mathrm{RB}$ in an aqueous phase, the maximum adsorption wavelength $\left(\lambda_{\max }\right)$ is identical (i.e., $\lambda_{\max }=550 \mathrm{~nm}$ ). The absorbance of DBwEECotton $_{100}$ at 400-480 $\mathrm{nm}$ is attributed to the presence of POP, which is consistent with the pale-yellow color of the SAFE-Cotton.

\subsection{Biocidal mechanisms of DBwEE-Cotton}

The ability to generate ROS under light exposure determines the biocidal function of the DBwEE-Cotton. The photoexcitation of a PS can be expressed by the Jablonski diagram shown in Fig. 2a. Specifically, the PS can absorb specific energy from the light and be excited to its singlet excited state $\left(S_{n}\right)$. With a unique chemical structure, the singlet excited state of the PS can efficiently convert to the triplet excited state $\left(\mathrm{T}_{1}\right)$, which exhibits a longer lifetime, through intersystem crossing (ISC). The $\mathrm{T}_{1}$ will then react with either hydrogen-donors (i.e., type I a)

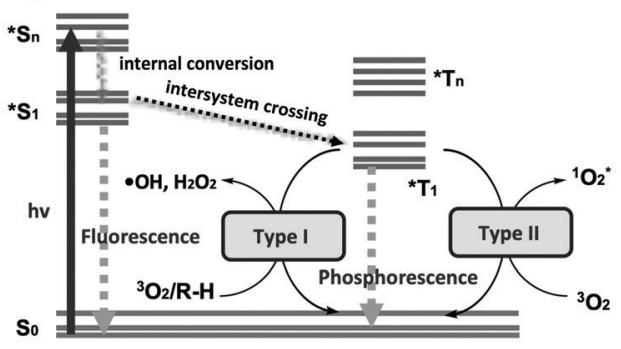

b)

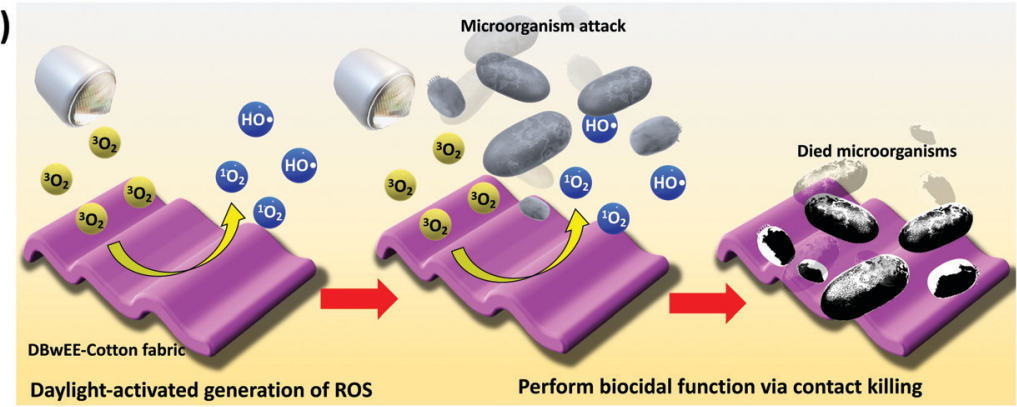

c)

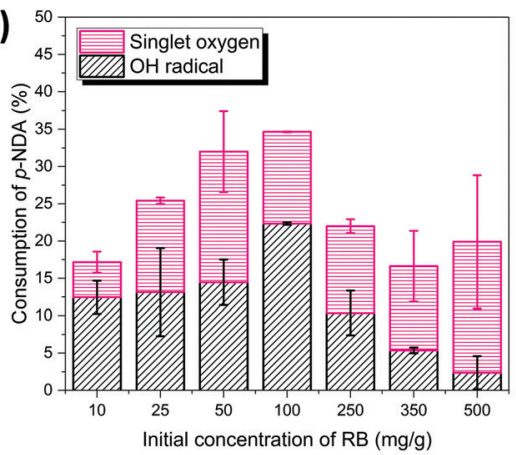

d)

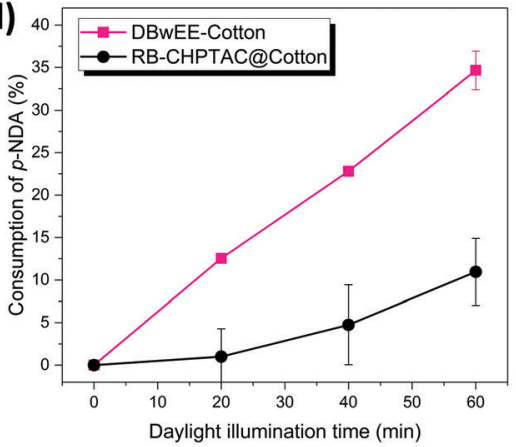

e)

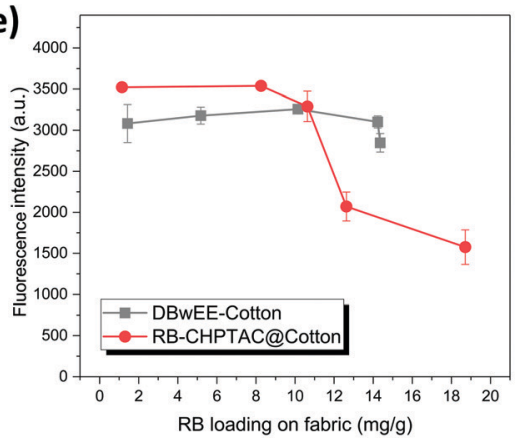

f)

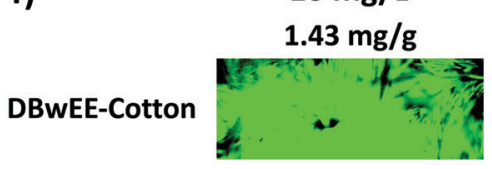

$50 \mathrm{mg} / \mathrm{L}$

$100 \mathrm{mg} / \mathrm{L}$ $5.18 \mathrm{mg} / \mathrm{g}$
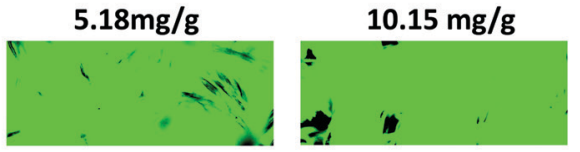

$250 \mathrm{mg} / \mathrm{L}$ $14.23 \mathrm{mg} / \mathrm{g}$ $500 \mathrm{mg} / \mathrm{L}$
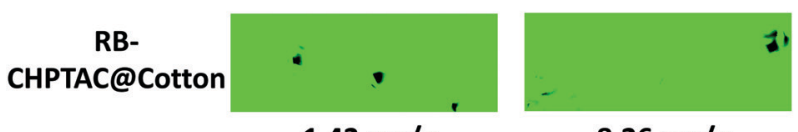

$8.26 \mathrm{mg} / \mathrm{g}$

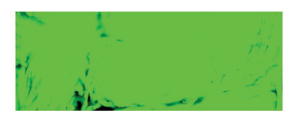

$10.63 \mathrm{mg} / \mathrm{g}$

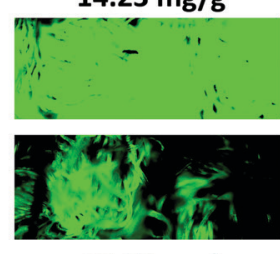

$12.63 \mathrm{mg} / \mathrm{g}$

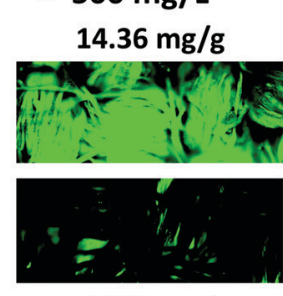

$18.71 \mathrm{mg} / \mathrm{g}$

Fig. 2 (a) Jablonski diagram of the excitation process of PSs. (b) Illustration of the biocidal mechanism of DBwEE-Cotton. (c) ROS production of DBwEE-Cotton under 30 min of daylight irradiation in relation to the initial concentration of RB. (d) ROS production of DBwEE-Cotton 100 and RB-CHPTAC (aCotton 100 . (e) Fluorescence intensities of DBwEE-Cotton and RB-CHPTAC (aCotton versus RB loading amounts. (f) Confocal fluorescence images of RB-dyed fabrics with labeled RB loading amounts. 
photoreaction) or oxygen (i.e., type II photoreaction) to generate ROS. With the presence of RB on the DBwEE-Cotton, the fabrics are expected to produce highly oxidative ROS under daylight irradiation, which resulted in biocidal activities against microorganisms. The bioprotective mechanism is illustrated in Fig. 2b.

$\mathrm{RB}$ is a well-known PS that possesses high efficiency in generating ${ }^{1} \mathrm{O}_{2}$ but with a very low possibility for type I photoreaction under daylight exposure. ${ }^{30}$ However, with the unique "posture" of RB molecules on the DBwEE-Cotton, i.e., RB was separately captured in mesopores that are enriched with secondary amines, the type I and type II photoreactions both occurred. The total production of ROS on the DBwEE-Cotton kept increasing on increasing the RB initial concentrations from $10 \mathrm{mg} \mathrm{L}^{-1}$ to $100 \mathrm{mg} \mathrm{L}^{-1}$, while it dropped when the $\mathrm{RB}$ concentration reached 250, 350 and $500 \mathrm{mg} \mathrm{L}^{-1}$ (Fig. 2c). In the later cases, the self-quenching of RB on the DBwEE-Cotton presented, which resulted from the RB aggregation in solutions with high concentration or indicated the presence of hydrophobic stacking of RB on the fabric with more RB loading. ${ }^{31}$ More interestingly, $\mathrm{HO}^{\bullet}$ was detected in the DBwEE-Cotton system other than solely ${ }^{1} \mathrm{O}_{2}$ when exposing to daylight. The production of $\mathrm{HO}^{\bullet}$ from DBwEE-Cotton ${ }_{100}$ was also checked by adding $10 \%$ DMSO (i.e., a $\mathrm{HO}^{\bullet}$ scavenger) in the $p$-NDA solution. Here, $p$-NDA was used as a colorimetric probe for selectively monitoring $\mathrm{HO}^{\bullet} .{ }^{12}$ After 30 min of daylight irradiation, the absorbance of $p$-NDA was unchanged when DMSO was presented, while $13.25 \%$ of $p$-NDA was consumed by $\mathrm{HO}^{\bullet}$ in the system without DMSO. This further proved the occurrence of type I photoreaction of RB on the DBwEE-Cotton. These results illustrated that the unique "posture" of RB on the DBwEE-Cotton not only enhanced the ${ }^{1} \mathrm{O}_{2}$ production but also modulated the photoreaction of RB. With the coexistence of both type I and type II photoreactions of RB on the DBwEE-Cotton, its biocidal activity can be further improved. The mechanisms of the enhanced biocidal efficiency of DBwEE-Cotton will be investigated based on two aspects: (1) separation of RB by mesopores, thus reducing the RB self-quenching; and (2) close position of RB with good hydrogen donors (i.e., POP), which forcedly achieves the type I photoreaction.

3.2.1 Alleviation of aggregation-caused self-quenching. First, the separation effect was investigated by fabricating another type of RB-dyed cotton fabric as a comparison. Specifically, cotton fabric was chemically modified by (2-chloro2-hydroxypropyl)-trimethylammonium chloride (CHPTAC) under an alkaline condition, which resulted in a monolayer of positive charges on the cotton fibers. The resulting material is denoted as CHPTAC@Cotton. The incorporation of the anionic RB on the fabric was achieved through electrostatic interaction with the cationic cotton. ${ }^{20}$ In this case, the selfquenching of the excited RB will be emphasized by having more aggregations of $\mathrm{RB}$ molecules on the fiber surfaces, thereby resulting in low ROS production. As shown in Fig. 2d, the ROS production between these two types of fabrics was significantly different. It is important to note that the RB amount on the CHPTAC@Cotton $_{100}\left(10.63 \mathrm{mg} \mathrm{g}^{-1}\right)$ was comparable to that on the DBwEE-Cotton ${ }_{100}\left(10.15 \mathrm{mg} \mathrm{g}^{-1}\right)$ (Fig. S2a and Table S2, $\mathrm{ESI} \dagger$ ). The total production of $\mathrm{ROS}$ (i.e., $\mathrm{HO}^{\bullet}$ and ${ }^{1} \mathrm{O}_{2}$ ) from the DBwEE-Cotton $_{100}$ after $60 \mathrm{~min}$ of daylight exposure was 3.4 times higher than that of the RB-CHPTA@Cotton ${ }_{100}$. And no type I photoreaction was found on the RB-CHPTA@Cotton (Fig. S5, ESI $†$ ). On the other hand, the photoexcitation efficiency can be examined by checking the fluorescence intensity of RB-embedded fabrics. The self-quenching of $\mathrm{S}_{n}$ states of RB promotes the radiation transition and leads to less fluorescence emission as well as low conversion to the $\mathrm{T}_{1}$ state (Fig. 2a). In Fig. 2e and $\mathrm{f}$, the variation of RB loading on the DBwEE-Cotton from $1.42 \mathrm{mg} \mathrm{g}^{-1}$ to $18.71 \mathrm{mg} \mathrm{g}^{-1}$ did not significantly affect the fluorescence emission, i.e., the fluorescence intensity swung around 3259 to 2847. In contrast, the RB-CHPTAC@Cotton dyed with low concentrations of $\mathrm{RB}\left(10,50\right.$, and $\left.100 \mathrm{mg} \mathrm{L}^{-1}\right)$ showed high fluorescence, while the intensity decreased dramatically when the RB loading reached $12.63 \mathrm{mg} \mathrm{g}^{-1}$ and $18.71 \mathrm{mg} \mathrm{g}^{-1}$. No big differences of the fluorescence intensity were showcased on DBwEE-Cotton and RB-CHPTAC@Cotton when the RB loading was lower than $10 \mathrm{mg} \mathrm{g}^{-1}$ of the fabric (Fig. 2f and e). However, the less efficient ROS production on the RB-CHPTAC@ Cotton $_{100}$ indicated that the self-quenching of the triplet excited state of $\mathrm{RB}\left({ }^{3} \mathrm{RB}^{*}\right)$ on the CHPTAC@Cotton is dominating over the potential paths of its singlet excited state $\left({ }^{1} \mathrm{RB}^{*}\right)$, since the ISC (quantum yield $\sim 1.0$ in water) ${ }^{30}$ is highly efficient over the internal conversion (IC) of ${ }^{1} \mathrm{RB}^{*}$ (Fig. 2d). However, the more significant self-quenching of ${ }^{1} \mathrm{RB}^{*}$ occurred once the $\mathrm{RB}$ on the RB-CHPTAC@Cotton reached to $12.63 \mathrm{mg} \mathrm{g}^{-1}$, with only $66.8 \%$ fluorescence retained to that of the DBwEE-Cotton 250 . The hydrophobic stacking between $\mathrm{RB}$ molecules on the RB-CHPTAC@Cotton ${ }_{500}$ with $18.71 \mathrm{mg} \mathrm{g}^{-1}$ of RB adsorbed presented more significant inhibition of the fluorescence emission. The over-loading of RB molecules on the DBwEE-Cotton can be avoided because of the guest-host adsorption mechanism. It clearly demonstrated that the avoidance of RB aggregation and dense stacking on a substrate is crucial for achieving photo-activity and consequently desired biocidal activity.

3.2.2 Type I photochemistry of RB. Secondly, the reason for $\mathrm{HO}^{\bullet}$ generation from the DBwEE-Cotton (i.e., a result of type I photoreaction) is interesting to be investigated. The occurrence of the type I reaction relates not only to the nature of the PS but also highly depends on the availability of hydrogen donors in the surrounding environment. The modulation of the type I mechanism over type II of RB was also noticed in an RB-lysozyme complex. ${ }^{30}$ Here, the capture of RB on the DBwEE-Cotton has created a unique environment where RB was surrounded by a large number of good H-donors, i.e., the secondary amines in the POP structure, at a very close distance. To get an insight into the favorability of $\mathrm{H}$-abstraction of $\mathrm{T}_{1} \mathrm{RB}$ from the POP, time-dependent density functional theory (TD-DFT) was applied to predict the Gibbs free energy changes $(\Delta G)$ of the reaction. In an aqueous system, POP contains positive charges by having the secondary amine being protonated, making it a much better $\mathrm{H}$-donor, and $\mathrm{RB}$ stays as anionic. As shown in Fig. 3a, the abstraction of a $\mathrm{H}$ from the POP by ${ }^{3} \mathrm{RB}^{*}$ led to the generation of a RB radical and POP radical, and 


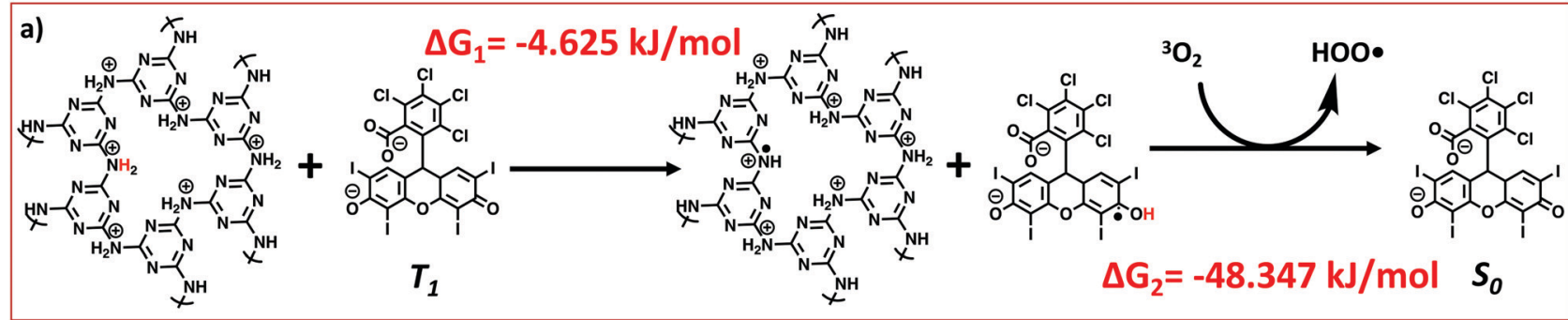

b)

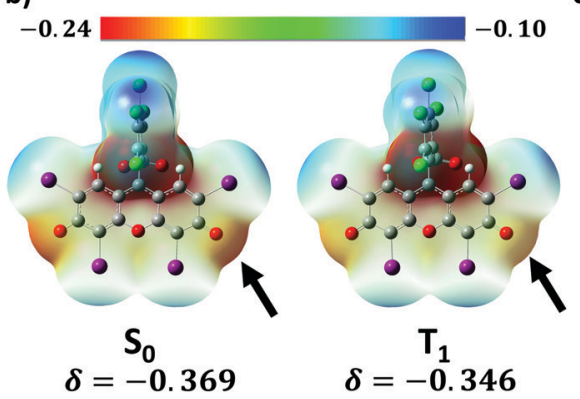

c)
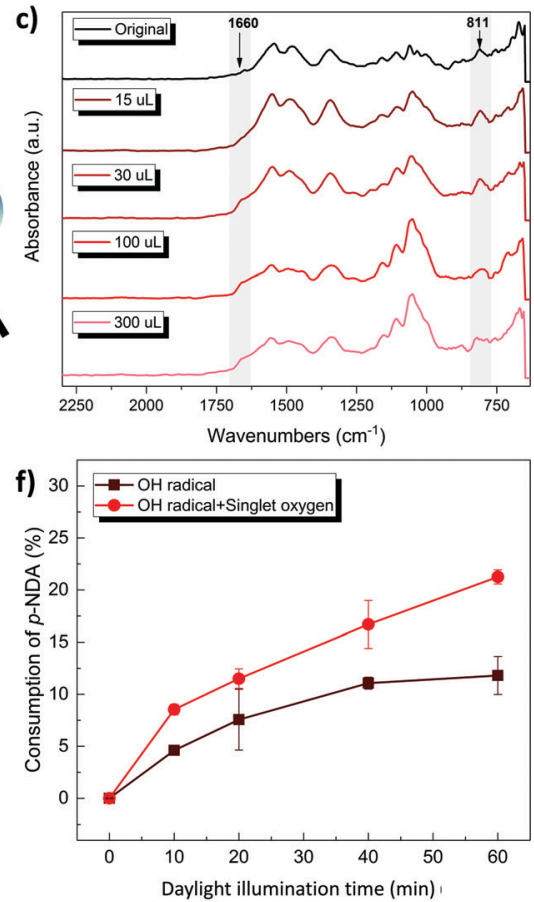
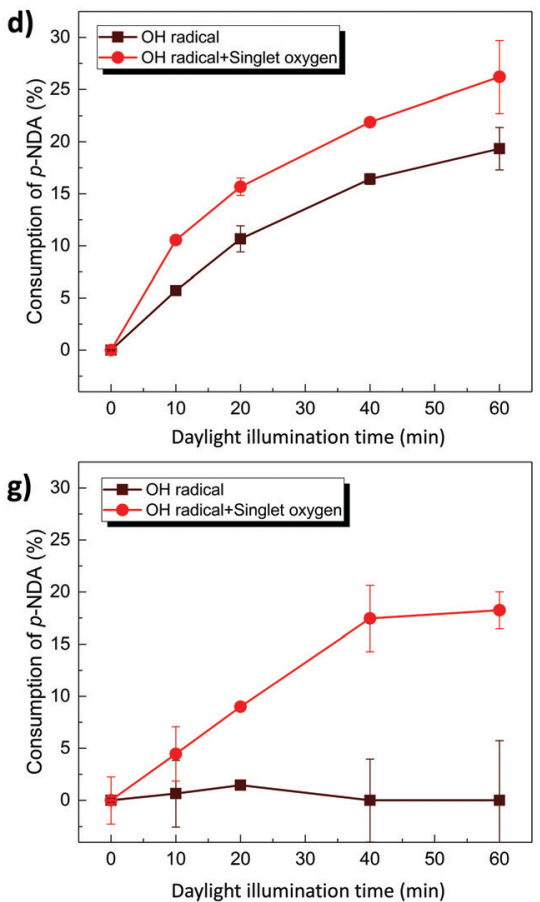

Fig. 3 (a) Gaussian calculated Gibbs free energy changes in the ROS production from the DBwEE-Cotton system. (b) Electrostatic potential map of RB at $\mathrm{S}_{0}$ and $\mathrm{T}_{1}$ states. (c) ATR-FTIR spectra of DBwEE-Cotton after different degrees of alkylation by Mel (i.e., contains different $\mathrm{H}$-donation abilities). ROS production of DBwEE-Cotton 100 after (d) $15 \mu \mathrm{L}$, (e) $30 \mu \mathrm{L}$, (f) $100 \mu \mathrm{L}$, and (g) $300 \mu \mathrm{L}$ of Mel alkylation for 24 hours.

this step was predicted to be spontaneous by showing a negative value of $\Delta G=-4.265 \mathrm{~kJ} \mathrm{~mol}^{-1}$. The following reaction of the $\mathrm{RB}$ radical with oxygen to generate $\mathrm{HOO}^{\bullet}$ was also spontaneous $\left(\Delta G=-48.347 \mathrm{~kJ} \mathrm{~mol}^{-1}\right)$, which illustrated the feasibility of the type I reaction that occurred in the DBwEECotton system. Moreover, the $\mathrm{H}$-abstraction tendency was checked with the electrostatic potential map of RB at $S_{0}$ and $\mathrm{T}_{1}$ states. As shown in Fig. $3 \mathrm{~b}$, the electron density of the carbonyl oxygen in the xanthene ring decreased when RB was excited from $\mathrm{S}_{0}(\delta=-0.369)$ to $\mathrm{T}_{1}(\delta=-0.346)$ states, illustrating the ability of ${ }^{3} \mathrm{RB}^{*}$ to undergo a $\mathrm{H}$-abstraction process.

To experimentally clarify the H-donor characteristic of POP, the secondary amines in its structure were blocked by treating the POP with methyl iodide (MeI) in different degrees, and the production amount of $\mathrm{HO}^{\bullet}$ was measured. In our previous study, the accumulated alkylation of the triazine-N of POP by MeI led to acidic- $\mathrm{H}$ elimination and electron redistribution, which converted secondary amines to imines (Fig. S6, ESI $\dagger$ ). ${ }^{27}$ In this case, this alkylation reaction is adopted to consume different amounts of H-donors in POP by controlling the MeI concentration. Specifically, 15, 30, 100, and $300 \mu \mathrm{L}$ of MeI were respectively incubated with DBwEE-Cotton ${ }_{100}$ for 24 hours, and then the chemical structure and the ROS production on the resultant fabrics were examined. As displayed in Fig. 3c, a shoulder peak at $1640 \mathrm{~cm}^{-1}$ gradually appeared on increasing the MeI concentrations, which refers to the formation of an imine structure in the alkylated POP. Moreover, the variation of the peak pattern around $800 \mathrm{~cm}^{-1}$ after reacting with high concentrations of $\mathrm{MeI}(e . g$., 100 and $300 \mu \mathrm{L})$ indicated the structural changes in the triazine rings. Based on the ROS production results, the loss of H-donors around the captured $\mathrm{RB}$ on the fabric was clearly shown. With fewer alkylations by MeI (e.g., 15 and $30 \mu \mathrm{L}$ ), the remaining H-donors were sufficient to trigger the type I photoreaction of $\mathrm{RB}$ under light exposure with high efficiency (Fig. 3d and e). However, the generation of $\mathrm{HO}^{\bullet}$ started to decrease as severe alkylation had occurred (e.g., treated by $100 \mu \mathrm{L}$ of MeI) (Fig. 3f), and the DBwEE-Cotton ${ }_{100}$ completely lost its $\mathrm{HO}^{\bullet}$ production ability after being treated by $300 \mu \mathrm{L}$ of $\mathrm{MeI}$, which only showed efficient ${ }^{1} \mathrm{O}_{2}$ generation (Fig. 3g). In summary, the presence of good H-donors, i.e., the secondary amines in POP, is the "secret" behind the performance of type I photoreaction of RB on the DBwEE-Cotton. 
a)

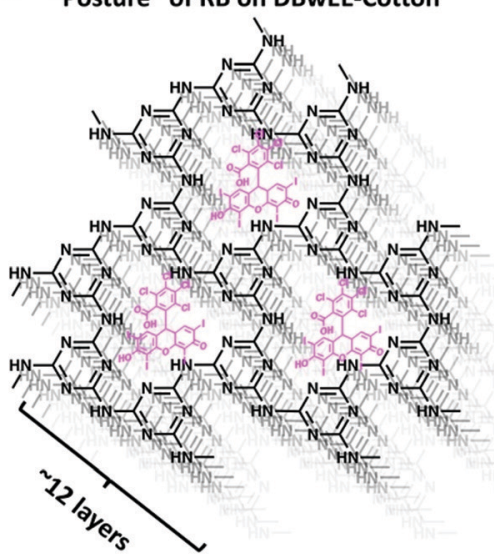
RB captured in POP on SAFE-Cotton efficiency

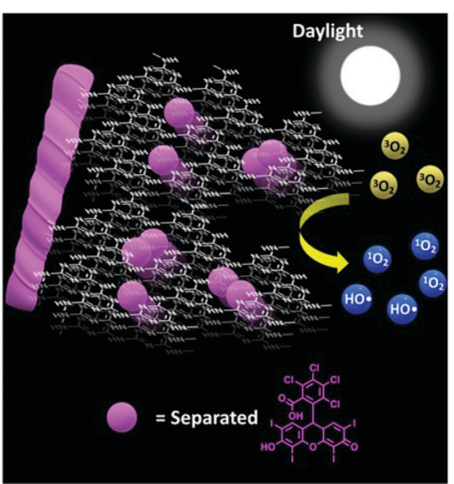

RB aggregated on CHPTAC@Cotton by electrostatic adsorption

Low ROS production and biocidal efficiency

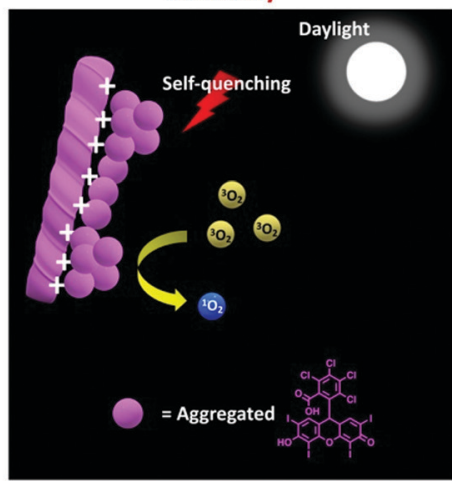

Fig. 4 (a) The "posture" of RB on DBwEE-Cotton. (b) Diagrammatic illustration of the mechanism of the enhanced ROS production efficiency of DBwEE-Cotton compared with that of the RB-CHPTACaCotton

The diagrammatic illustration of the RB "postures" and ROS production mechanisms of DBwEE-Cotton and RB-CHPTAC@ Cotton are summarized in Fig. 4. Under the daylight irradiation, the captured RB inside the mesopores of the DBwEE-Cotton avoided the aggregation-caused self-quenching and forcefully triggered the occurrence of type I photoreaction of $\mathrm{RB}$, thus resulting in high ROS production and biocidal efficiency. In contrast, the RB molecules were adsorbed on the CHPTAC@ Cotton by electrostatic interaction and densely packed via hydrophobic interaction between each RB molecule, leading to the selfquenching of RB and showcased less efficient biocidal function.

\subsection{Antibacterial function of DBwEE-Cotton}

After carefully investigating the mechanism of the enhanced biocidal function of DBwEE-Cotton, the antibacterial properties of the fabrics (i.e., DBwEE-Cotton ${ }_{100}$ ) were examined by challenging them with both Gram-negative (i.e., E. coli) and Gram-positive (i.e., L. innocua) bacteria with exposure to daylight in an XL-1500 crosslinker box for specific durations (e.g., $5,10,20,30$, and $60 \mathrm{~min})$. The pristine cotton $\left(2 \times 2 \mathrm{~cm}^{2}\right)$ was contaminated by the bacteria and exposed to light for $60 \mathrm{~min}$ as the control. All the bacterial reductions were calculated based on the bacteria count in the pristine cotton samples. As showcased in Fig. 5a and b, 99.9999\% of E. coli and L. innocua were effectively killed within 20 min under daylight exposure, which is much more efficient than other traditional biocidal textiles (Table S3, ESI $\dagger$ ). It is also exciting to notice that the DBwEE-Cotton $_{100}$ rendered $99 \%$ and $99.99 \%$ of bacterial reduction against both Gram-negative and Gram-positive bacteria with only 5 and 10 min of light exposure, respectively.
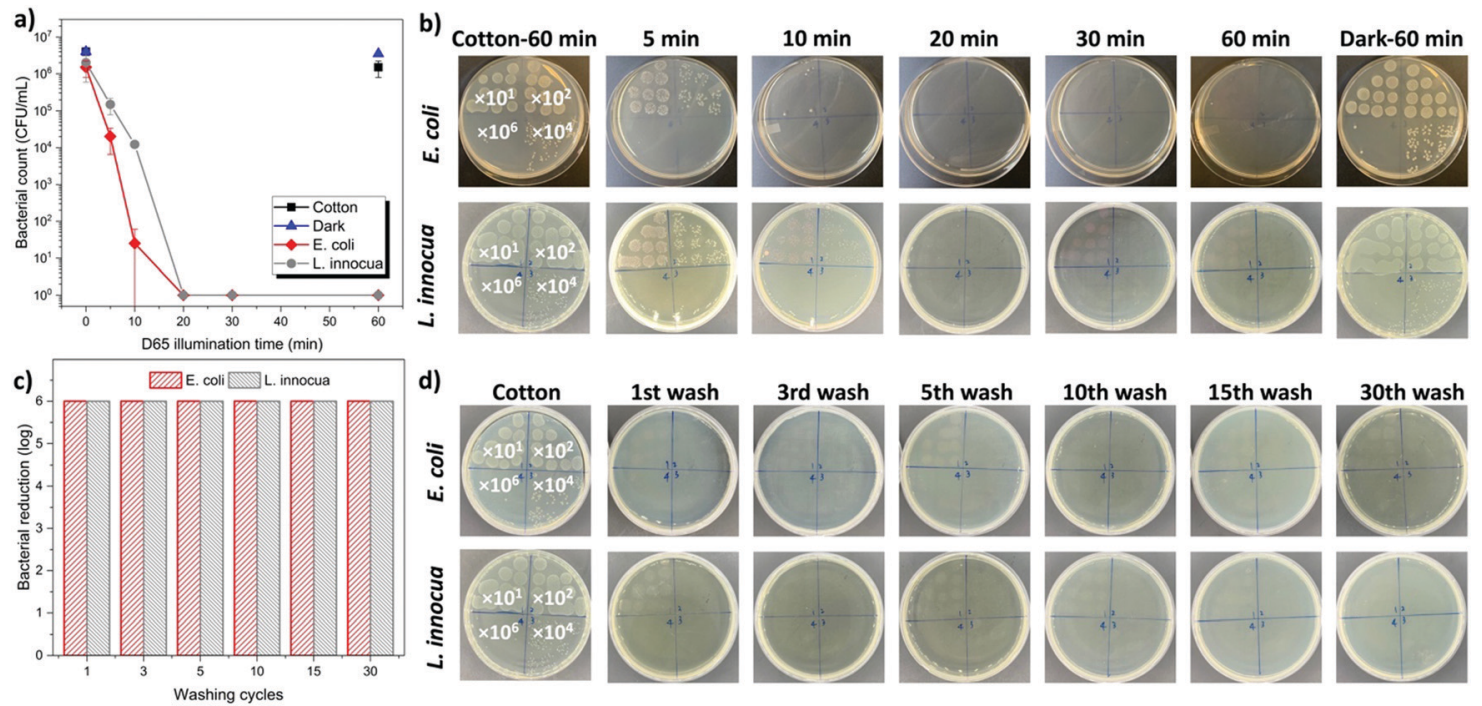

Fig. 5 (a) Antibacterial results of DBwEE-Cotton 100 against E. coli and L. innocua. (b) Images of agar plates corresponding to different treatment conditions. (c) Antibacterial efficiency of DBwEE-Cotton 100 against $E$. coli and L. innocua under daylight irradiation for 30 min after different wash cycles. (d) Images of agar plates corresponding to DBwEE-Cotton 100 after different washing cycles. 
This rapid bioprotective function of DBwEE-Cotton ensured instant and sufficient protection against lethal pathogens.

The washing durability of the DBwEE-Cotton is another crucial factor for its repeated and long-term usage. The fabrics (i.e., DBwEE-Cotton ${ }_{100}$ ) were washed with water containing $0.15 \mathrm{wt} \%$ of AATCC standard detergent at $40{ }^{\circ} \mathrm{C}$ for $45 \mathrm{~min}$, which counted as one cycle of washing according to the AATCC Test Method 61-2007. Antibacterial tests were performed on the DBwEE-Cotton $_{100}$ after 1, 3, 5, 10, 15, and 30 times of washes, and the results are displayed in Fig. $5 \mathrm{c}$ and d. After $30 \mathrm{~min}$ of daylight exposure, E. coli $(6 \log )$ and L. innocua $(6 \log )$ were completely inactivated by the fabrics, even after 30 washes. This proved that the electrostatic interaction and the guest-host capture of RB on the DBwEE-Cotton greatly benefit the washing durability of the fabrics. On the other hand, the light stability of the DBwEE-Cotton ${ }_{100}$ was evaluated by bacterial challenges after exposing the fabric to an office light (light intensity = 3000 lux) for six days. Neither E. coli nor L. innocua stayed alive on the DBwEE-Cotton Doo $_{100}$ after 30 min of daylight irradiation, which demonstrated the feasibility of DBwEE-Cotton ${ }_{100}$ for long-term applications (Fig. S7, ESI $\dagger$ ).

\section{Conclusions}

We designed and demonstrated a unique "posture" of RB on cotton fabrics containing POP, with biocidal activity being significantly enhanced against both Gram-negative (i.e., E. coli) and Gram-positive (i.e., L. innocua) bacteria. Based on the capture of RB molecules separately in the mesopores of SAFECotton, the aggregation-caused self-quenching of $\mathrm{RB}$ on the solid support highly diminished. Moreover, the RB on the DBwEE-Cotton was found to undergo both type I and type II photoreactions, thus further improving the biocidal efficiency by producing more ROS for pathogen killing. The occurrence of the type I photoreaction of RB was forced to occur in the POP system, which was realized by closely surrounding RB molecules with massive good H-donors (i.e., POP). As a result, the DBwEE-Cotton ${ }_{100}$ presented highly improved biocidal functions based on the contact killing mechanism. More than 99.9999\% of $E$. coli and L. innocua were disinfected within 20 min under daylight exposure. The DBwEE-Cotton ${ }_{100}$ also exhibited excellent washing durability (i.e., $6 \log$ of bacterial reduction after 30 washes) and light stability (i.e., $6 \log$ of bacterial reduction after six days of light exposure). The successful design of DBwEE-Cotton is expected to inspire the development of novel PPE with aggressive protection accompanied by enhanced efficiency, washability, robustness, and long-term applicability.

\section{Author contributions}

P. Tang: conceptualization, formal analysis, investigation, methodology, validation and writing - original draft; A. Y. El-Moghazy: investigation and methodology; B. Ji: investigation, methodology and writing - review \& editing; N. Nitin: resource and supervision; G. Sun: conceptualization, visualization, supervision and writing - review \& editing.

\section{Conflicts of interest}

The authors claimed no conflict of interest.

\section{Acknowledgements}

This work was financially supported by the COVID-19 Research Accelerator Funding Track Program (fund source number 19933) at the University of California, Davis, CA, USA, the California Department of Pesticide Regulation, USA (18-C0012), and the National Institute of Environmental Health Sciences (NIEHS), USA (Grant No. 5P42ES004699). The authors would like to thank the Advanced Material Characterization and Testing Lab (AMCaT) and the Campus Mass Spectrometry Facility at the University of California Davis, CA, USA for performing the SEM imaging (The ThermoFisher Quattro ESEM was funded through the US National Science Foundation, Grant No. DMR-1725618) and MALDI-TOF-MS, respectively.

\section{References}

1 C. J. E. Metcalf and J. Lessler, Science, 2017, 357, 149-152.

2 M. D. Snape and R. M. Viner, Science, 2020, 370, 286-288.

3 S. E. Eikenberry, M. Mancuso, E. Iboi, T. Phan, K. Eikenberry, Y. Kuang, E. Kostelich and A. B. Gumel, Infect. Dis. Model., 2020, 5, 293-308.

4 V. C. C. Cheng, S. C. Wong, V. W. M. Chuang, S. Y. C. So, J. H. K. Chen, S. Sridhar, K. K. W. To, J. F. W. Chan, I. F. N. Hung, P. L. Ho and K. Y. Yuen, J. Infect., 2020, 81, 107-114.

5 G. Liu, J. Xiang, Q. Xia, K. Li, H. Yan and L. Yu, Ind. Eng. Chem. Res., 2020, 59, 9666-9678.

6 Q. B. Xu, W. S. Zheng, P. P. Duan, J. N. Chen, Y. Y. Zhang, F. Y. Fu, H. Y. Diao and X. D. Liu, Carbohydr. Polym., 2019, 204, 42-49.

7 M. Zahid, E. L. Papadopoulou, G. Suarato, V. D. Binas, G. Kiriakidis, I. Gounaki, O. Moira, D. Venieri, I. S. Bayer and A. Athanassiou, ACS Appl. Bio Mater., 2018, 1, 1154-1164.

8 Y. Ma, J. Li, Y. Si, K. Huang, N. Nitin and G. Sun, ACS Appl. Mater. Interfaces, 2019, 11, 17814-17822.

9 C. Huang, Y. Chen, G. Sun and K. Yan, Materials, 2019, $12,127$.

10 F. Wang, J. Dai, L. Huang, Y. Si, J. Yu and B. Ding, ACS Nano, 2020, 14, 8975-8984.

11 Y. Si, Z. Zhang, W. Wu, Q. Fu, K. Huang, N. Nitin, B. Ding and G. Sun, Sci. Adv., 2018, 4(3), eaar5931.

12 Z. Zhang, Y. Si and G. Sun, ACS Sustainable Chem. Eng., 2019, 7, 18493-18504.

13 W. Chen, J. Chen, L. Li, X. Wang, Q. Wei, R. A. Ghiladi and Q. Wang, ACS Appl. Mater. Interfaces, 2019, 11, 29557-29568. 
14 X. Nie, S. Wu, A. Mensah, Q. Wang, F. Huang, D. Li and Q. Wei, J. Colloid Interface Sci., 2020, 579, 233-242.

15 F. C. Fang, Nat. Rev. Microbiol., 2004, 2, 820-832.

16 L. Hu, A. Hou, K. Xie and A. Gao, ACS Appl. Mater. Interfaces, 2019, 11, 26500-26506.

17 Z. Zhang, A. Y. El-Moghazy, N. Wisuthiphaet, N. Nitin, D. Castillo, B. G. Murphy and G. Sun, ACS Appl. Mater. Interfaces, 2020, 12, 49416-49430.

18 K. H. Hong and G. Sun, J. Appl. Polym. Sci., 2010, 115, 1138-1144.

19 T. Zhang, H. Yu, J. Li, H. Song, S. Wang, Z. Zhang and S. Chen, Mater. Today Phys., 2020, 15, 100254.

20 P. Tang, Z. Zhang, A. Y. El-Moghazy, N. Wisuthiphaet, N. Nitin and G. Sun, ACS Appl. Mater. Interfaces, 2020, 12, 49442-49451.

21 P. Fini, R. Loseto, L. Catucci, P. Cosma and A. Agostiano, Bioelectrochemistry, 2007, 70, 44-49.

22 M. Mirenda, C. A. Strassert, L. E. Dicelio and E. S. Román, ACS Appl. Mater. Interfaces, 2010, 2, 1556-1560.
23 K. Liu, Y. Liu, Y. Yao, H. Yuan, S. Wang, Z. Wang and X. Zhang, Angew. Chem., Int. Ed., 2013, 52, 8285-8289.

24 D. Bagchi, A. Bhattacharya, T. Dutta, S. Nag, D. Wulferding, P. Lemmens and S. K. Pal, ACS Appl. Bio Mater., 2019, 2, 1772-1780.

25 C. Li, F. Lin, W. Sun, F. G. Wu, H. Yang, R. Lv, Y. X. Zhu, H. R. Jia, C. Wang, G. Gao and Z. Chen, ACS Appl. Mater. Interfaces, 2018, 10, 16715-16722.

26 S. Qian, L. Song, L. Sun, X. Zhang, Z. Xin, J. Yin and S. Luan, J. Photochem. Photobiol., A, 2020, 400, 112626.

27 P. Tang, B. Ji and G. Sun, J. Mater. Chem. A, 2020, 24128-24136.

28 P. Tang and G. Sun, Carbohydr. Polym., 2017, 160, 153-162. 29 S. K. Kundu and A. Bhaumik, RSC Adv. , 2015, 5, 32730-32739. 30 E. Fuentes-Lemus, M. Mariotti, P. Hägglund, F. Leinisch, A. Fierro, E. Silva, C. López-Alarcón and M. J. Davies, Free Radical Biol. Med., 2019, 143, 375-386.

31 D. Xu and D. C. Neckers, J. Photochem. Photobiol., A, 1987, 40, 361-370. 